\title{
Editorial
}

\section{Advanced Green Energy Nanomaterials for Optoelectronic Devices: Synthesis, Processing, Characterization, and Applications}

\author{
Wei-Chun Chen, ${ }^{1}$ Shou-Yi Kuo, ${ }^{2,3}$ Ying-Lung Daniel Ho, ${ }^{4}$ \\ Kaushik Balakrishnan, ${ }^{5}$ and Wei-Heng Shih ${ }^{6}$ \\ ${ }^{1}$ Instrument Technology Research Center, National Applied Research Laboratories, Hsinchu City, Taiwan \\ ${ }^{2}$ Department of Electronic Engineering, Chang Gung University, Taoyuan, Taiwan \\ ${ }^{3}$ Department of Nuclear Medicine, Chang Gung Memorial Hospital, Taoyuan, Taiwan \\ ${ }^{4}$ Department of Electrical and Electronic Engineering, University of Bristol, Bristol, UK \\ ${ }^{5}$ College of Optical Sciences, University of Arizona, Tucson, AZ, USA \\ ${ }^{6}$ Department of Materials Science and Engineering, Drexel University, Philadelphia, PA, USA
}

Correspondence should be addressed to Wei-Chun Chen; weichun@narlabs.org.tw

Received 1 September 2016; Accepted 1 September 2016

Copyright (C) 2016 Wei-Chun Chen et al. This is an open access article distributed under the Creative Commons Attribution License, which permits unrestricted use, distribution, and reproduction in any medium, provided the original work is properly cited.

Advanced green energy nanomaterials in the applied science fields have received increasing attentions because of their unique physical and chemical properties. Their dominant roles are established in our science and society and their applications penetrate into our daily life and the influence impact is significant. This special issue aims at identifying the current status and anticipating future directions in this emerging field via fusions of civil engineering, environmental science, chemistry, and materials science and antiecotoxic technologies. With the unique structures and novel properties of the nanomaterials, the applications cover a wide range of key components or products, such as light-emitting diodes (LED), solar cell devices, nanocomposites, optical materials, functional materials, and other related advanced nanomaterials.

Five research articles were accepted in this special issue, including papers on synthesis of functional nanomaterials and analysis, synthesis of nitride for LEDs, optimized TTP processes to CNT-based materials, and nanocrystalline nitrides growth. For synthesis of nanomaterials, the papers indicated that the properties of the nanocrystalline TiZrN thin films changed with nitrogen flow rate above $1.0 \mathrm{sccm}$ because the films contained a stable single phase (TiZrN).

For thin films growth, the papers are about the thin films and their physical properties. One tried to describe how AZO films can be used as the transparent conducting oxide thin films. In addition, $\mathrm{ZnO}$ thin films were deposited by CVAD at room temperature for flexible devices. For the optoelectronic performance of GaN-based light-emitting diodes devices, fabrication of FTP-PSSs is helpful in improving the crystal quality of GaN epilayer.

We hope that this special issue provides the readers with an overview of the recent progress achieved and the future developing directions in the advanced green energy nanomaterials synthesis technology. This special issue will be a valuable reference and a perspective for the research community working in this exciting field and can inspire more engineers and scientists.

Wei-Chun Chen Shou-Yi Kuo Ying-Lung Daniel Ho Kaushik Balakrishnan Wei-Heng Shih 

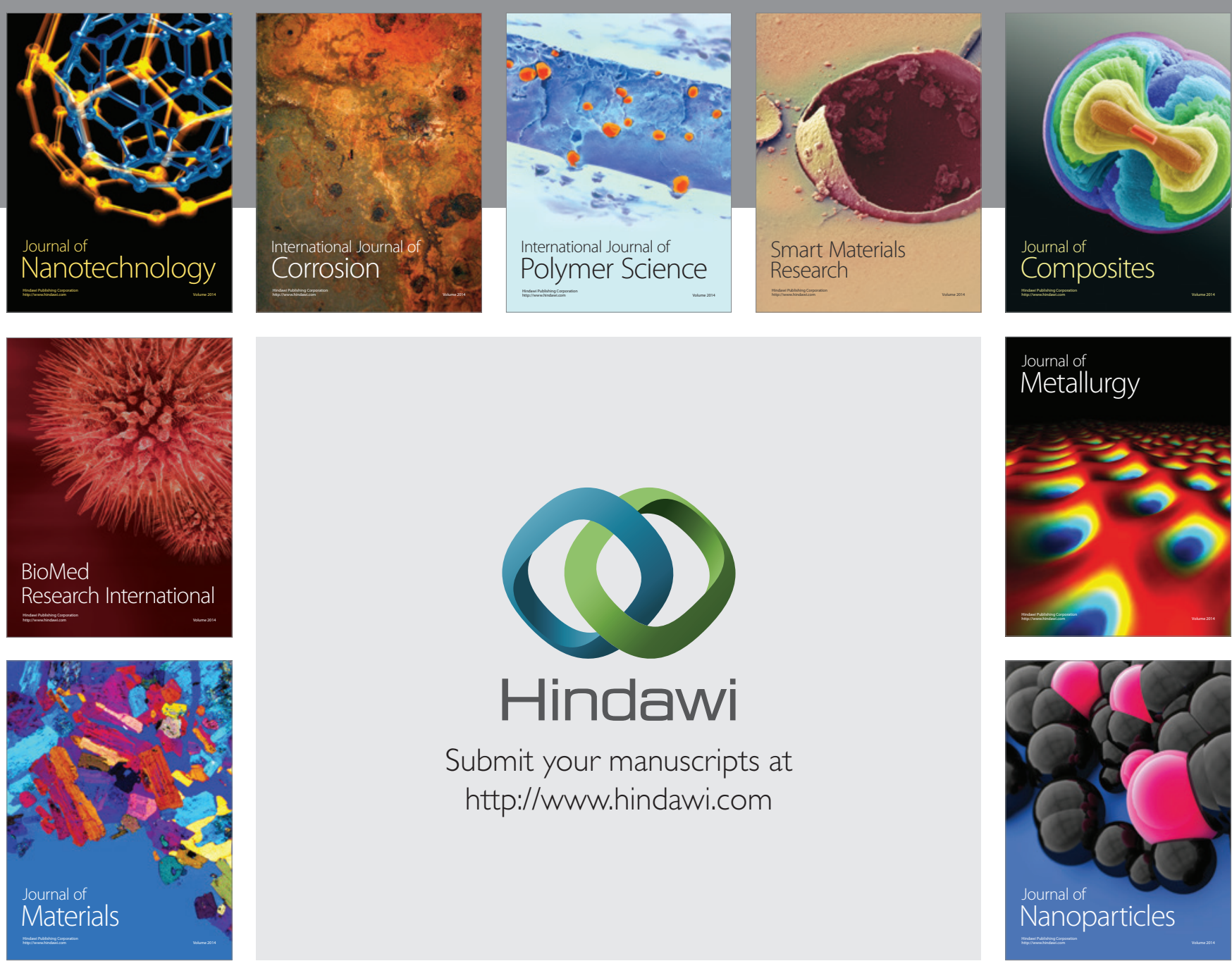

\section{Hindawi}

Submit your manuscripts at

http://www.hindawi.com

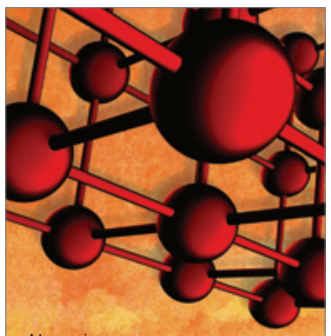

Materials Science and Engineering
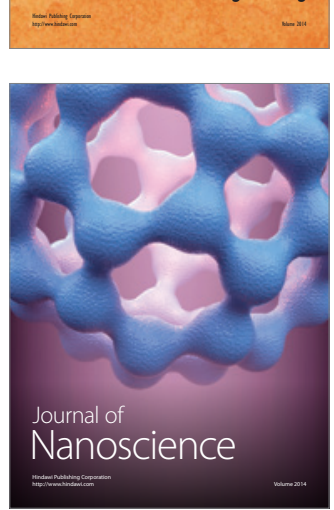
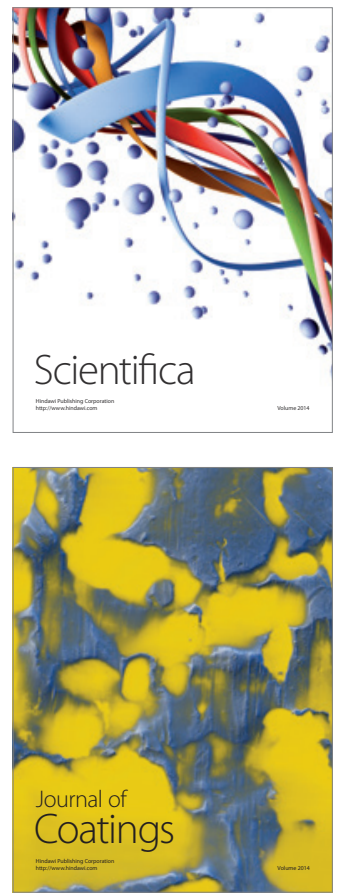
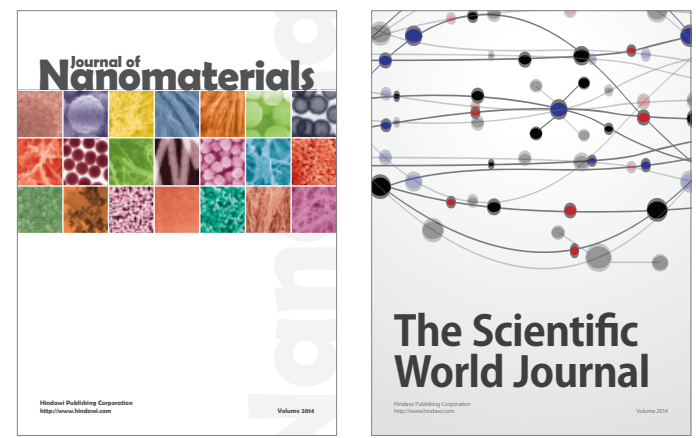

The Scientific World Journal
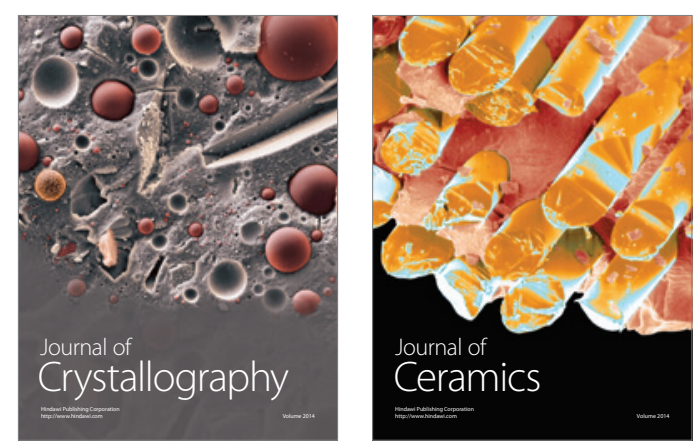
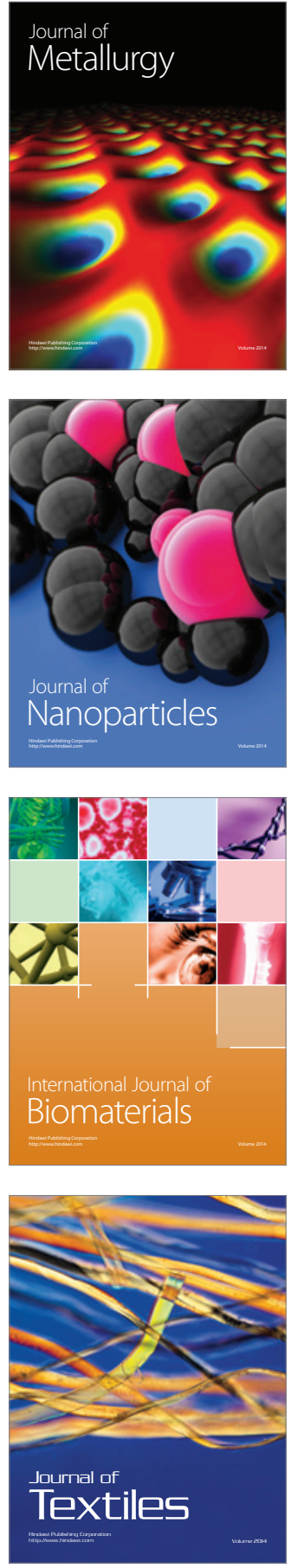